University of Nebraska - Lincoln

DigitalCommons@University of Nebraska - Lincoln

M. Eugene Rudd Publications

Research Papers in Physics and Astronomy

2-22-1996

\title{
New Model for Electron-Impact Ionization Cross Sections of Molecules
}

W. Hwang

National Institute of Standards and Technology, Gaithersburg, Maryland 20899

Y.-K. Kim

National Institute of Standards and Technology, Gaithersburg, Maryland 20899

M. Eugene Rudd

University of Nebraska - Lincoln, erudd@unl.edu

Follow this and additional works at: https://digitalcommons.unl.edu/physicsrudd

Part of the Physics Commons

Hwang, W.; Kim, Y.-K.; and Rudd, M. Eugene, "New Model for Electron-Impact Ionization Cross Sections of Molecules" (1996). M. Eugene Rudd Publications. 21.

https://digitalcommons.unl.edu/physicsrudd/21

This Article is brought to you for free and open access by the Research Papers in Physics and Astronomy at DigitalCommons@University of Nebraska - Lincoln. It has been accepted for inclusion in M. Eugene Rudd Publications by an authorized administrator of DigitalCommons@University of Nebraska - Lincoln. 
J. Chem. Phys. / Volume 104 / Issue 8

Issue Date: 22 February 1996

\title{
New model for electron-impact ionization cross sections of molecules
}

\author{
J. Chem. Phys. 104, 2956 (1996); DOI:10.1063/1.471116 \\ W. Hwang and Y.-K. Kim \\ National Institute of Standards and Technology, Gaithersburg, Maryland 20899
}

M. E. Rudd

Department of Physics and Astronomy, University of Nebraska-Lincoln, Lincoln, Nebraska 68588-0111

\begin{abstract}
A theoretical model for electron-impact ionization cross sections, which has been developed primarily for atoms and atomic ions, is applied to neutral molecules. The new model combines the binary-encounter theory and the Bethe theory for electron-impact ionization, and uses minimal theoretical data for the ground state of the target molecule, which are readily available from public-domain molecular structure codes such as GAMESS. The theory is called the binaryencounter Bethe (BEB) model, and does not, in principle, involve any adjustable parameters. Applications to 19 molecules, including $\mathrm{H}_{2}, \mathrm{NO}, \mathrm{CH}_{2}, \mathrm{C}_{6} \mathrm{H}_{6}$, and $\mathrm{SF}_{6}$, are presented, demonstrating that the BEB model provides total ionization cross sections by electron impact from threshold to several keV with an average accuracy of $15 \%$ or better at the cross section peak, except for $\mathrm{SiF}_{3}$. The BEB model can be applied to stable molecules as well as to transient radicals.

History: Received 13 September 1995; accepted 15 November 1995

Permalink: http://link.aip.org/link/?JCPSA6/104/2956/1

Keywords: cross sections, electron-, molecule collisions, ground states, ionization, nitrogen oxides, wave functions, hydrogen molecules, impact phenomena

Copyright (C1996 American Institute of Physics.
\end{abstract}




\title{
New model for electron-impact ionization cross sections of molecules
}

\author{
W. Hwang ${ }^{\text {a) }}$ and Y.-K. Kim \\ National Institute of Standards and Technology, Gaithersburg, Maryland 20899 \\ M. E. Rudd \\ Department of Physics and Astronomy, University of Nebraska-Lincoln, Lincoln, Nebraska 68588-0111
}

(Received 13 September 1995; accepted 15 November 1995)

\begin{abstract}
A theoretical model for electron-impact ionization cross sections, which has been developed primarily for atoms and atomic ions, is applied to neutral molecules. The new model combines the binary-encounter theory and the Bethe theory for electron-impact ionization, and uses minimal theoretical data for the ground state of the target molecule, which are readily available from public-domain molecular structure codes such as GAMESS. The theory is called the binary-encounter Bethe (BEB) model, and does not, in principle, involve any adjustable parameters. Applications to 19 molecules, including $\mathrm{H}_{2}, \mathrm{NO}, \mathrm{CH}_{2}, \mathrm{C}_{6} \mathrm{H}_{6}$, and $\mathrm{SF}_{6}$, are presented, demonstrating that the BEB model provides total ionization cross sections by electron impact from threshold to several keV with an average accuracy of $15 \%$ or better at the cross section peak, except for $\mathrm{SiF}_{3}$. The $\mathrm{BEB}$ model can be applied to stable molecules as well as to transient radicals. (C) 1996 American Institute of Physics. [S0021-9606(96)01708-X]
\end{abstract}

\section{INTRODUCTION}

Although there are several useful theories for electronimpact ionization cross sections for atoms and atomic ions, few of them are extendable to neutral molecules and molecular ions, primarily because it is difficult to calculate molecular continuum wave functions, which most of these theories require. In addition, the collision of slow incident electrons with a target requires an approach that treats the incident electron and the bound electrons in the target (or at least the electron being ejected) on equal footing as a compound system, for instance, by introducing strong coupling and exchange interaction between them. This is one of the major reasons for the failure of most theories, particularly those based on the perturbation approach, at low incident electron energies. At high incident electron energies, the plane-wave Born approximation provides accurate ionization cross sections when used with reliable initial- and final-state wave functions.

Strong coupling theories which treat the colliding system as a compound system, such as the close-coupling or the $R$-matrix method, require a large basis set to describe the system, making it very difficult to treat ionizing collisions. Moreover, strong coupling theories tend to produce a large number of resonances, both real and virtual, which may have to be averaged over for practical applications, such as in the modeling of plasma chemistry and radiation effects. Except for a very recent theoretical method called the convergent close-coupling method, ${ }^{1}$ these strong coupling theories are mostly limited to discrete excitations and difficult to extend to ionization.

In this article, we describe a new theoretical method that provides reliable electron-impact ionization cross sections for molecules using very simple input data, all of which can

\footnotetext{
a)Present address: Ultraprecision Technology Team, Samsung Electronics Co., Suwon, Korea.
}

be obtained from standard molecular wave function codes for the ground state of a molecule. There are no adjustable or fitted parameters in our theory. Our method does not provide details of resonances in the continuum, vibrational and/or rotational excitations concomitant with ionization, multiple ionization, dissociative ionization, etc. It simply predicts the total ionization cross section as the sum of ionization cross sections for ejecting one electron from each of the atomic or molecular orbital. We will show that it is valid over the entire energy range, from the first ionization threshold up to several $\mathrm{keV}$ in incident electron energies.

As is outlined in Sec. II, our theory combines the Mott cross section ${ }^{2}$ modified by the binary-encounter theory ${ }^{3}$ for low incident energies $T$ with the Bethe theory ${ }^{4}$ for high $T$. Many models have been proposed to use this combination, ${ }^{5-8}$ but they all require either some empirical parameters or explicit knowledge of the continuum dipole oscillator strengths of the target molecule or its constituent atoms. Some of these models require empirical parameters which are difficult to obtain, or use a large number of such parameters. Our model uses a new way to determine the ratio between the low- $T$ and high- $T$ cross sections without using any empirical parameters, and ionization cross sections are derived from analytic expressions for the entire range of $T$ with three molecular constants per molecular orbital, which are available from molecular structure codes. Deep inner shells do not contribute appreciably to total ionization cross sections; hence they can be omitted in most theories for total ionization cross sections including ours.

Because of its simplicity, our theory can predict cross sections for complex molecules such as $\mathrm{C}_{3} \mathrm{H}_{8}$ and $\mathrm{SF}_{6}$ as easily as for simple ones such as $\mathrm{H}_{2}$. Moreover, our theory can be applied not only to stable molecules but also to transient radicals. The applicability of our theory is limited only by the availability of a molecular structure code that can provide basic information on molecular orbitals. Judging 
from the examples presented in this article and our experience with other atoms and molecules, our theory can provide total ionization cross sections accurate enough to be used in modeling of plasma chemistry, magnetic fusion plasmas, and radiation effects.

Two other theoretical methods to estimate ionization cross sections with comparable flexibility are the "DM approach" based on an additivity rule developed by Deutsch et al. ${ }^{6}$ and the Weizsäcker-Williams method ("WW method") as modified by Seltzer. ${ }^{8}$ The DM approach constructs a molecular ionization cross section by adding ionization cross sections for the constituent atoms. The basic shape of these atomic cross sections is given by the classical theory of Gryzinski, ${ }^{9}$ while their absolute values are given in terms of (a) atomic orbital radii, which can be obtained from atomic wave function codes, (b) atomic orbital occupation numbers, which are derived from the Mulliken population analysis of the target molecule, and (c) atomic weighting factors, which have been fitted to known atomic ionization cross sections. Our theory uses far fewer, ab initio molecular parameters which are also standard output of molecular structure codes. Although the BEB model does not require any experimental data since theoretical values of binding energy $B$ and kinetic energy $U$ are available, we prefer to use the experimental value for the ionization potential of the outermost electron to obtain the correct threshold for comparison to experiments. The threshold behavior of ionization cross sections is sensitive to the value of the lowest electron binding energy (not the dissociation energy!). The WW method $^{8}$ is very similar to our BED model described in the next section in that it requires explicit data on continuum dipole oscillator strengths of the target. However, the WW method is primarily designed for high-energy incident electrons, and may lead to unrealistic results for slow incident electrons of hundreds of $\mathrm{eV}$ or lower.

The underlying theory of our model is outlined in Sec. II, application examples are described in Sec. III, and conclusions are presented in Sec. IV.

\section{OUTLINE OF THEORY}

Recently, we proposed the binary-encounter-dipole (BED) model for electron-impact ionization cross sections of atoms and molecules. ${ }^{10}$ This BED model combines the binary-encounter theory ${ }^{3}$ and the Bethe theory. ${ }^{4}$ The ratio between the binary-encounter theory and the Bethe theory is set by requiring the asymptotic form at high incident energy $T$ of the former to match that of the latter both in the ionization cross section and in the stopping cross section. The stopping cross section, which is the integral of the product of the energy-loss cross section and the energy loss of the incident electron, is used to evaluate the stopping power of the target medium. The BED model provides a formula to calculate the singly differential cross section, or the energy distribution of ejected electrons $d \sigma / d W$ with the ejected electron energy $W$, for each atomic or molecular orbital. To apply the BED model, one needs for each orbital the electron binding energy $B$, the average kinetic energy $U=\left\langle\mathbf{p}^{2} / 2 m\right\rangle$ with the bound electron momentum $\mathbf{p}$ and its mass $m$, the orbital occupation number $N$ and the continuum dipole oscillator strength $d f / d W$.

The value of the kinetic energy $U$ for each orbital in the initial state (usually the ground state) of the target is a theoretical quantity evaluated in any atomic or molecular wave function code that calculates the total energy. However, both the initial- and continuum-state wave functions are needed to calculate $d f / d W$ and this is the only nontrivial data needed to apply the BED model. Alternatively, $d f / d W$ can be deduced from experimental photoionzation cross sections, though partial cross sections are needed to deduce $d f / d W$ for each orbital. The total ionization cross section $\sigma_{i}$ is then obtained by integrating $d \sigma / d W$ over the allowed range of $W$, i.e., from 0 to $(T-B) / 2$

$\sigma_{i}(t)=\frac{S}{t+u+1}\left[D(t) \ln t+\left(2-\frac{N_{i}}{N}\right)\left(\frac{t-1}{t}-\frac{\ln t}{t+1}\right)\right]$,

where $t=T / B, u=U / B, S=4 \pi a_{0}^{2} N R^{2} / B^{2}, a_{0}=0.5292 \AA$, $R=13.61 \mathrm{eV}$,

$$
D(t) \equiv N^{-1} \int_{0}^{(t-1) / 2} \frac{1}{w+1} \frac{d f(w)}{d w} d w,
$$

with $w=W / B$, and

$$
N_{i} \equiv \int_{0}^{\infty} \frac{d f(w)}{d w} d w .
$$

The BED model was found to be very effective in reproducing known values of $d \sigma / d W$ and $\sigma_{i}$ for small atoms and molecules, demonstrating an agreement of $\pm 10 \%$ or better for the entire range of incident electron energies in most cases. $^{10}$

Although one can in principle calculate $d f / d W$ for each orbital, it is available only for a limited number of atoms and very few molecules. Hence, we also proposed a simplified version of the BED theory when no information on $d f / d W$ is available. In this case, which we refer to as the binaryencounter-Bethe (BEB) model, ${ }^{10}$ we assume a simple form for $d f / d W$,

$$
\frac{d f}{d w}=N /(w+1)^{2}
$$

such that the integrated cross section $\sigma_{\mathrm{BEB}}$ per orbital is given by

$$
\sigma_{\mathrm{BEB}}=\frac{S}{t+u+1}\left[\frac{\ln t}{2}\left(1-\frac{1}{t^{2}}\right)+1-\frac{1}{t}-\frac{\ln t}{t+1}\right] .
$$

Equation (4) approximates the shape of $d f / d W$ for the ionization of the ground state of $\mathrm{H}$ and generates reliable differential ionization cross sections only for the targets with simple shell structures, ${ }^{10}$ such as $\mathrm{H}, \mathrm{He}$, and $\mathrm{H}_{2}$.

In Eqs. (1) and (5), the term associated with the first logarithmic function on the right-hand side (RHS) represents distant collisions (large impact parameters) dominated by the dipole interaction, and the rest of the terms on the RHS represent close collisions (small impact parameters) as described by the Mott cross section. The second logarithmic 
TABLE I. Molecular orbitals, electron binding energy $B$ in eV, kinetic energy $U$ in eV, and electron occupation number $N$ for $\mathrm{H}_{2}, \mathrm{~N}_{2}, \mathrm{O}_{2}, \mathrm{CO}, \mathrm{NO}$, $\mathrm{H}_{2} \mathrm{O}, \mathrm{CO}_{2}$, and $\mathrm{NH}_{3}$. All $B$ and $U$ values are theoretical, except for those marked by an asterisk, which are experimental.

\begin{tabular}{|c|c|c|c|c|}
\hline Molecule & MO & $B$ & $U$ & $N$ \\
\hline $\mathrm{H}_{2}$ & $1 \sigma_{g}$ & $15.43^{*}$ & 15.98 & 2 \\
\hline \multirow[t]{4}{*}{$\mathrm{N}_{2}$} & $2 \sigma_{g}$ & 41.72 & 71.13 & 2 \\
\hline & $2 \sigma_{u}^{\circ}$ & 21.00 & 63.18 & 2 \\
\hline & $1 \pi_{u}$ & 17.07 & 44.30 & 4 \\
\hline & $3 \sigma_{g}$ & $15.58^{*}$ & 54.91 & 2 \\
\hline \multirow{5}{*}{$\begin{array}{c}\mathrm{O}_{2} \text {, triplet } \\
\text { Average of } \alpha \text { and } \beta \text { orbital values }\end{array}$} & $2 \sigma_{g}$ & 46.19 & 79.73 & 2 \\
\hline & $2 \sigma_{u}$ & 29.82 & 90.92 & 2 \\
\hline & $1 \pi_{u}$ & 19.64 & 59.89 & 4 \\
\hline & $3 \sigma_{g}$ & 19.79 & 71.84 & 2 \\
\hline & $1 \pi_{g}^{s}$ & $12.07 *$ & 84.88 & 2 \\
\hline \multirow[t]{4}{*}{$\mathrm{CO}$} & $3 \sigma$ & 41.92 & 79.63 & 2 \\
\hline & $4 \sigma$ & 21.92 & 73.18 & 2 \\
\hline & $1 \pi$ & 17.66 & 54.30 & 4 \\
\hline & $5 \sigma$ & $14.01 *$ & 42.26 & 2 \\
\hline NO, doublet & $3 \sigma$ & 43.70 & 76.55 & 2 \\
\hline \multirow{4}{*}{ Average of $\alpha$ and $\beta$ orbital values } & $4 \sigma$ & 25.32 & 77.04 & 2 \\
\hline & $1 \pi$ & 18.49 & 55.37 & 4 \\
\hline & $5 \sigma$ & $15.87^{*}$ & 62.25 & 2 \\
\hline & $2 \pi$ & $9.26^{*}$ & 65.27 & 1 \\
\hline \multirow[t]{4}{*}{$\mathrm{H}_{2} \mathrm{O}$} & $2 a_{1}$ & 36.88 & 70.71 & 2 \\
\hline & $1 b_{2}$ & 19.83 & 48.36 & 2 \\
\hline & $3 a_{1}$ & 15.57 & 59.52 & 2 \\
\hline & $1 b_{1}$ & $12.61^{*}$ & 61.91 & 2 \\
\hline \multirow[t]{6}{*}{$\mathrm{CO}_{2}$} & $3 \sigma_{1 g}$ & 42.04 & 75.72 & 2 \\
\hline & $2 \sigma_{2 u}$ & 40.60 & 78.38 & 2 \\
\hline & $4 \sigma_{1 g}$ & 21.62 & 74.66 & 2 \\
\hline & $3 \sigma_{2 u}$ & 20.27 & 71.56 & 2 \\
\hline & $1 \pi_{u}$ & 19.70 & 49.97 & 4 \\
\hline & $1 \pi_{g}$ & $13.77^{*}$ & 64.43 & 4 \\
\hline \multirow[t]{3}{*}{$\mathrm{NH}_{3}$} & $2 a_{1}$ & 31.13 & 48.49 & 2 \\
\hline & $1 e$ & 17.19 & 35.62 & 4 \\
\hline & $3 a_{1}$ & $10.16^{*}$ & 43.25 & 2 \\
\hline
\end{tabular}

function originates from the interference of the direct and exchange scattering also described by the Mott cross section.

We present the values of $B, U$, and $N$ for small molecules in Table I, those for hydrocarbons in Table II, and the data for $\mathrm{SiF}_{x}, x=1-3$, and $\mathrm{SF}_{6}$ in Table III. The data for $\mathrm{H}_{2}$ are from the correlated wavefunction of Kołos and Roothaan, ${ }^{11}$ while the rest of the data in Tables I-III are from the molecular structure code GAMESS. ${ }^{12}$ Since deep inner shells, such as the $K$ shells of $\mathrm{N}_{2}$ and $\mathrm{O}_{2}$, contribute little to total ionization cross sections, we have omitted them from the tables, though we included them to calculate BEB cross sections for small molecules.

One can use either theoretical or experimental values of $B$, while $U$ is a theoretical quantity that cannot be directly measured, though the sum of all $U$ 's is equal to the total energy of the target molecule according to the virial theorem. Since experimental values of $B$ are often smaller than theoretical ones, the $\mathrm{BEB}$ cross sections obtained using experimental $B$ values are usually higher (by $10 \%-15 \%$ at the
TABLE II. Molecular orbitals, electron binding energy $B$ in eV, kinetic energy $U$ in $\mathrm{eV}$, and electron occupation number $N$ for hydrocarbons. All $B$ and $U$ values are theoretical, except for those marked by an asterisk, which are experimental.

\begin{tabular}{|c|c|c|c|c|}
\hline Molecule & $\mathrm{MO}$ & $B$ & $U$ & $N$ \\
\hline \multirow{4}{*}{$\begin{array}{c}\mathrm{CH}_{2} \text {, triplet } \\
\text { Average of } \alpha \text { and } \beta \text { orbital values }\end{array}$} & $2 a_{1}$ & 23.59 & 35.20 & 2 \\
\hline & $1 b_{1}$ & 16.43 & 26.70 & 2 \\
\hline & $3 a_{1}$ & 12.32 & 31.88 & 1 \\
\hline & $1 b_{2}$ & $10.40^{*}$ & 31.80 & 1 \\
\hline \multirow{3}{*}{$\begin{array}{c}\mathrm{CH}_{3} \text {, doublet } \\
\text { Average of } \alpha \text { and } \beta \text { orbital values }\end{array}$} & $2 a_{1}$ & 24.57 & 34.18 & 2 \\
\hline & $1 e$ & 15.64 & 26.46 & 4 \\
\hline & $3 a_{1}$ & $9.84^{*}$ & 30.40 & 1 \\
\hline \multirow[t]{2}{*}{$\mathrm{CH}_{4}$} & $2 a_{1}$ & 25.73 & 33.05 & 2 \\
\hline & $1 t_{2}$ & $12.51^{*}$ & 25.96 & 6 \\
\hline \multirow{6}{*}{$\mathrm{C}_{2} \mathrm{H}_{4}$} & $2 a_{g}$ & 28.23 & 40.97 & 2 \\
\hline & $2 b_{2 u}^{s}$ & 21.56 & 33.49 & 2 \\
\hline & $1 b_{3 u}$ & 17.55 & 25.33 & 2 \\
\hline & $3 a_{g}$ & 16.08 & 35.00 & 2 \\
\hline & $1 b_{1 g}^{\circ}$ & 13.74 & 28.56 & 2 \\
\hline & $1 b_{1 u}$ & $10.51^{*}$ & 26.51 & 2 \\
\hline \multirow[t]{5}{*}{$\mathrm{C}_{2} \mathrm{H}_{6}$} & $2 a_{1 g}$ & 27.75 & 34.37 & 2 \\
\hline & $2 a_{2 u}$ & 22.99 & 33.60 & 2 \\
\hline & $1 e_{u}$ & 16.31 & 24.42 & 4 \\
\hline & $3 a_{1 g}$ & 13.90 & 32.78 & 2 \\
\hline & $1 e_{g}$ & $11.52^{*}$ & 28.17 & 4 \\
\hline \multirow[t]{10}{*}{$\mathrm{C}_{3} \mathrm{H}_{8}$} & $3 a_{1}$ & 28.69 & 34.45 & 2 \\
\hline & $2 b_{1}$ & 25.25 & 34.96 & 2 \\
\hline & $4 a_{1}$ & 21.86 & 33.32 & 2 \\
\hline & $1 b_{2}$ & 17.04 & 23.46 & 2 \\
\hline & $5 a_{1}$ & 16.30 & 25.80 & 2 \\
\hline & $3 b_{1}$ & 15.03 & 28.08 & 2 \\
\hline & $1 a_{2}$ & 14.53 & 27.09 & 2 \\
\hline & $6 a_{1}$ & 12.98 & 31.21 & 2 \\
\hline & $4 b_{1}$ & 12.97 & 34.09 & 2 \\
\hline & $2 b_{2}$ & $10.95^{*}$ & 28.53 & 2 \\
\hline \multirow[t]{10}{*}{$\mathrm{C}_{6} \mathrm{H}_{6}$} & $2 a_{1 g}$ & 31.38 & 39.32 & 2 \\
\hline & $2 e_{1 u}$ & 27.64 & 42.43 & 4 \\
\hline & $2 e_{2 g}$ & 22.42 & 39.00 & 4 \\
\hline & $3 a_{1 g}$ & 19.38 & 25.38 & 2 \\
\hline & $2 b_{1 u}$ & 17.50 & 34.21 & 2 \\
\hline & $1 b_{2 u}$ & 16.86 & 40.02 & 2 \\
\hline & $3 e_{1 u}$ & 16.03 & 32.58 & 4 \\
\hline & $1 a_{2 u}$ & 13.67 & 23.90 & 2 \\
\hline & $3 e_{2 g}$ & 13.45 & 37.96 & 4 \\
\hline & $1 e_{1 g}$ & $9.25^{*}$ & 28.27 & 4 \\
\hline
\end{tabular}

cross section peak) than those obtained using theoretical $B$ values. Using the experimental value for the lowest electron binding energy (=first ionization potential) will not only assure that the cross section starts at the right threshold but also we found that the shape and magnitude of the BEB cross section near the threshold agree better with known experimental cross sections. On the other hand, the cross sections with theoretical values of $B$ tend to agree better with experiment near the peak $(T \sim 100 \mathrm{eV})$.

For closed-shell molecules, we used the restricted Hartree-Fock (RHF) method with the default Gaussian basis set (known as the 6-311-G set) provided by the GAMESS code. For open-shell molecules, we found that the unre- 
TABLE III. Molecular orbitals, electron binding energy $B$ in eV, kinetic energy $U$ in $\mathrm{eV}$, and electron occupation number $N$ for $\mathrm{SiF}_{x}, x=1-3$, and $\mathrm{SF}_{6}$. All $B$ and $U$ values are theoretical, except for those marked by an asterisk, which are experimental. Atomic orbitals which contribute $\geqslant 90 \%$ of molecular charge density are identified in parentheses.

\begin{tabular}{|c|c|c|c|c|}
\hline Molecule & MO & $B$ & $U$ & $N$ \\
\hline \multirow{8}{*}{$\begin{array}{c}\mathrm{SiF}, \text { quartet } \\
\text { average of } \alpha \text { and } \beta \text { orbital values }\end{array}$} & $3 \sigma(\mathrm{Si} 2 s)$ & 168.63 & 359.53 & 2 \\
\hline & $1 \pi($ Si $2 p)$ & 117.15 & 332.44 & 4 \\
\hline & $4 \sigma(\operatorname{Si} 2 p)$ & 117.18 & 331.55 & 2 \\
\hline & $5 \sigma$ & 42.84 & 104.41 & 2 \\
\hline & $6 \sigma$ & 19.99 & 74.82 & 2 \\
\hline & $2 \pi$ & 17.84 & 81.34 & 4 \\
\hline & $7 \sigma(\mathrm{Si} 3 s)$ & 16.30 & $55.09 / 3$ & 1 \\
\hline & $3 \pi(\operatorname{Si} 3 p)$ & $7.28 *$ & $34.39 / 3$ & 2 \\
\hline \multirow[t]{13}{*}{$\mathrm{SiF}_{2}$} & $3 a_{1}(\mathrm{Si} 2 s)$ & 169.13 & 360.19 & 2 \\
\hline & $2 b_{1}(\mathrm{Si} 2 p)$ & 117.52 & 331.76 & 2 \\
\hline & $4 a_{1}(\mathrm{Si} 2 p)$ & 117.47 & 332.52 & 2 \\
\hline & $1 b_{2}(\operatorname{Si} 2 p)$ & 117.47 & 331.38 & 2 \\
\hline & $5 a_{1}(\mathrm{~F} 2 s)$ & 43.89 & 102.17 & 2 \\
\hline & $3 b_{1}(\mathrm{~F} 2 s)$ & 43.22 & 106.55 & 2 \\
\hline & $6 a_{1}$ & 21.56 & 80.10 & 2 \\
\hline & $4 b_{1}$ & 19.53 & 84.66 & 2 \\
\hline & $7 a_{1}$ & 18.97 & 77.67 & 2 \\
\hline & $2 b_{2}$ & 18.60 & 77.19 & 2 \\
\hline & $1 a_{2}(\mathrm{~F} 2 p)$ & 17.75 & 83.62 & 2 \\
\hline & $5 b_{1}(\mathrm{~F} 2 p)$ & 17.36 & 88.13 & 2 \\
\hline & $8 a_{1}(\mathrm{Si} 3 s)$ & $10.78^{*}$ & $46.19 / 3$ & 2 \\
\hline \multirow{12}{*}{$\begin{array}{c}\mathrm{SiF}_{3} \text {, doublet } \\
\text { average of } \alpha \text { and } \beta \text { orbital values }\end{array}$} & $3 a_{1}(\operatorname{Si} 2 s)$ & 170.32 & 359.73 & 2 \\
\hline & $4 a_{1}(\operatorname{Si} 2 p)$ & 118.79 & 332.26 & 2 \\
\hline & $2 e(\operatorname{Si} 2 p)$ & 118.85 & 331.83 & 4 \\
\hline & $5 a_{1}$ & 44.75 & 101.00 & 2 \\
\hline & $3 e$ & 43.95 & 106.28 & 4 \\
\hline & $6 a_{1}$ & 22.57 & 81.11 & 2 \\
\hline & $4 e$ & 20.47 & 82.42 & 4 \\
\hline & $7 a_{1}$ & 19.71 & 75.76 & 2 \\
\hline & $5 e$ & 19.03 & 82.66 & 4 \\
\hline & $6 e(\mathrm{~F})$ & 18.29 & 85.84 & 4 \\
\hline & $1 a_{2}(\mathrm{~F})$ & 17.86 & 88.76 & 2 \\
\hline & $8 a_{1}(\mathrm{Si} 3 s)$ & $9.3^{*}$ & $52.38 / 3$ & 1 \\
\hline \multirow[t]{12}{*}{$\mathrm{SF}_{6}$} & $3 a_{1 g}(\mathrm{~S} 2 s)$ & 256.18 & 510.35 & 2 \\
\hline & $2 t_{1 u}(\mathrm{~S} 2 p)$ & 193.27 & 479.25 & 6 \\
\hline & $4 a_{1 g}$ & 50.93 & 85.62 & 2 \\
\hline & $3 t_{1 u}$ & 47.09 & 101.6 & 6 \\
\hline & $2 e_{g}$ & 45.50 & 110.3 & 4 \\
\hline & $5 a_{1 g}^{g}$ & 30.32 & 98.55 & 2 \\
\hline & $4 t_{1 u}$ & 25.61 & 83.20 & 6 \\
\hline & $1 t_{2 g}$ & 23.03 & 75.55 & 6 \\
\hline & $3 e_{g}$ & 20.35 & 86.84 & 4 \\
\hline & $1 t_{2 u}(\mathrm{~F})$ & 20.06 & 90.74 & 6 \\
\hline & $5 t_{1 u}$ & 19.69 & 91.23 & 6 \\
\hline & $1 t_{1 g}(\mathrm{~F})$ & $15.33^{*}$ & 98.29 & 6 \\
\hline
\end{tabular}

stricted Hartree-Fock (UHF) method produced more realistic orbital energies-which we took as the electron binding energies as prescribed by the Koopman theorem-for valence orbitals than the restricted open-shell Hartree-Fock (ROHF) method. Although the UHF method has the disadvantage of producing "too many" orbital and kinetic energies $-\alpha$ and $\beta$ orbitals - the valence orbital energies from the ROHF method were often unrealistically small, making the corresponding cross section too large, sometimes by as much as $50 \%$ at the cross section peak. On the other hand, using $B$ and $U$ values from the UHF method produces cross sections almost identical to those produced using the average between the $B$ and $U$ values from the matching $\alpha$ and $\beta$ orbitals. The $B$ and $U$ values presented in Tables I-III are these average values for open-shell molecules.

Deducing experimental $B$ values for inner orbitals is not straightforward for molecules, particularly when the outermost orbital is only partially occupied, in addition to the ambiguity of whether to use the "vertical" or "relaxed" binding energies. In practice, the BEB model is insensitive to minor variations in the values of $B$ and $U$ used, except for the lowest $B$. For these reasons, we have used the experimental values for the lowest $B$, which are available for many molecules and radicals, ${ }^{13}$ and theoretical values for the remaining orbitals.

Most $B$ values listed in Tables I-III are slightly different from those quoted in Rudd et al. ${ }^{14}$ because the experimental $B$ values were quoted in the latter, while we used mostly theoretical values in the present work. Also, some $U$ values in Ref. 14-e.g., for $\mathrm{NH}_{3}, \mathrm{H}_{2} \mathrm{O}$-are too high because they were not divided by the electron occupation numbers, or in some cases-e.g., for $\mathrm{SF}_{6}$ and $\mathrm{TeF}_{6}$ - too low because pseudopotentials were used. The inner-shell molecular orbitals from pseudopotentials lack nodes in the core region, leading to unrealistically low $U$ values. Minor differences in the $U$ values resulted also from the use of different molecular wave function codes in the present work and Ref. 14. We recommend the values in Tables I-III.

\section{APPLICATIONS TO MOLECULES}

In this section, we compare our theoretical cross sections, mostly BEB but some BED cross sections, to a large number of molecules. Most older experiments measured the "gross" ionization cross section, which is determined by measuring the total ion current rather than the number of ions. On the other hand, most theoretical values are the "counting" ionization cross section, which accounts for the number of ions produced. When many multiply charged ions are produced, the gross ionization cross section will be significantly larger than the counting ionization cross section. The cross sections based on the BEB and BED models are counting ionization cross sections, and therefore should be considered as the lower limits to experimental gross ionization cross sections. In modern experiments, both molecular ions as well as their fragments are often collected using mass spectrometers. Since the BEB and BED cross sections are simple sums of cross sections for ejecting one electron from each molecular orbital, the theory cannot give a detailed account of dissociative ionization or fragments produced. Hence, comparisons of the theory with experiments on large molecules with diverse channels for dissociative ionization and fragmentation are not straightforward. For simplicity, we compared our theoretical cross sections to the simple sum of all experimental partial cross sections that produced an ion. Nevertheless, the comparisons presented here will clearly demonstrate the utility of our theory, which is applicable to a wide range of molecules. 


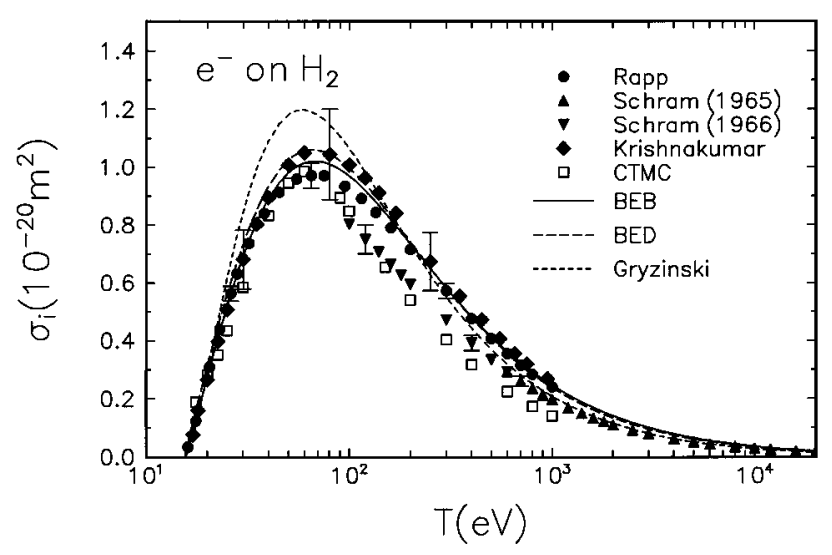

FIG. 1. Comparison of the BED and BEB cross sections to other theory and experiment for $\mathrm{H}_{2}$. Solid curve, the BEB cross section; long-dashed curve, the BED cross section (Ref. 10); short-dashed curve, classical theory (Ref. 9); squares, CTMC theory (Ref. 19); circles, experimental data by Rapp and Englander-Golden (Ref. 15); triangles, data by Schram et al. (Ref. 17); inverted triangles, Schram et al. (Ref. 18); diamonds, data by Krishnakumar and Srivastava (Ref. 16).

\section{A. Diatomic molecules}

In Fig. 1, we compare our BEB cross section for $\mathrm{H}_{2}$ to the experimental data by Rapp and Englander-Golden, ${ }^{15}$ the data by Krishnakumar and Srivastava, ${ }^{16}$ those by Schram et al. ${ }^{17,18}$ the BED cross section in which accurate experimental $d f / d W$ was used, ${ }^{10}$ classical cross section by Gryzinski, ${ }^{9}$ and the classical trajectory Monte Carlo (CTMC) cross section by Schultz et al. ${ }^{19}$ The BED cross section in Fig. 1 is slightly higher than that shown in Fig. 7 of Ref. 10 because the $U$ value used there was wrong. The correct value is given in Table I. The classical theory of Gryzinski ${ }^{9}$ tends to overestimate the peak value not only of $\mathrm{H}_{2}$ but also of other targets, such as $\mathrm{H}$ and $\mathrm{N}_{2} \cdot{ }^{10,20}$

Although the BEB model seems to agree slightly better with the cross section by Rapp and Englander-Golden than the BED model at the peak, the latter will definitely provide better differential cross sections, particularly for more complex targets where electron correlation strongly affects the $d f / d W$ of valence shells. The high accuracy $( \pm 4.5 \%)$ claimed by Rapp and Englander-Golden implies that the excellent agreement between the BED cross section and the data by Krishnakumar and Srivastava near the cross section peak is accidental. The CTMC cross section at high $T$ falls short of experimental values because the CTMC theory lacks the dipole contribution, which dominates at high $T$. The experimental data by Schram et al. are too low despite the high accuracy $( \pm 6.7 \%)$ claimed by the authors.

Existing experimental photoionization data of $\mathrm{N}_{2}$ were analyzed earlier ${ }^{14}$ and the $d f / d W$ for outer molecular orbitals are available. These data were used to derive the BED cross section, Eq. (1), for $\mathrm{N}_{2}$. The BED and BEB cross sections for $\mathrm{N}_{2}$ are compared in Fig. 2 to the experimental data by Rapp and Englander-Golden, ${ }^{15}$ the data by Schram et al. ${ }^{17,18}$ the data by Krishnakumar and Srivastava,${ }^{21}$ and very recent experimental data by Straub et al. ${ }^{22}$ The experimental uncer-

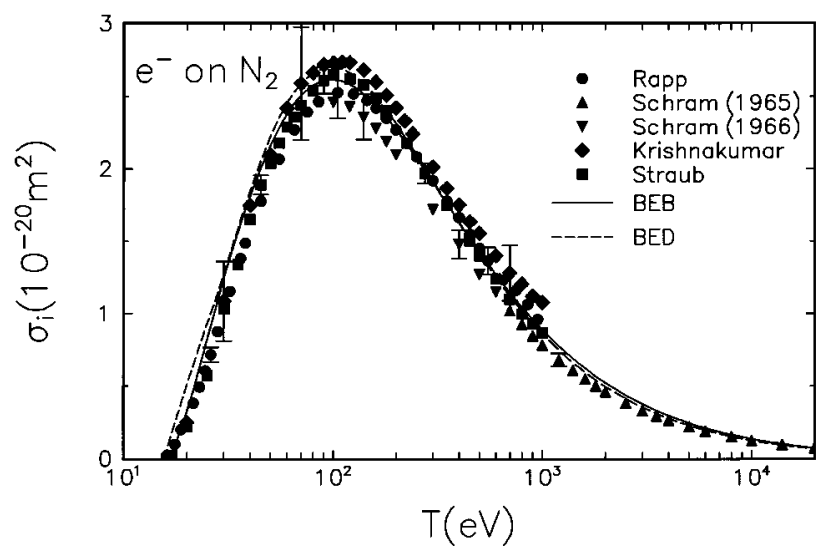

FIG. 2. Comparison of the BED and BEB cross sections to experiment for $\mathrm{N}_{2}$. Solid curve, the BEB cross section; dashed curve, the BED cross section; circles, experimental data by Rapp and Englander-Golden (Ref. 15); triangles, data by Schram et al. (Ref. 17); inverted triangles, data by Schram et al. (Ref. 18); diamonds, Krishnakumar and Srivastava (Ref. 21); squares, data by Straub et al. (Ref. 22).

tainty of the data by Straub et al., $\pm 3.5 \%$, is the smallest among the data cited in this article, while the uncertainty of the data by Rapp and Englander-Golden is $\pm 7 \%$, and that of the data by Krishnakumar and Srivastava is $\pm 15 \%$. As in the case of $\mathrm{H}_{2}$, the BEB cross section agrees with the experiments better than the BED cross section in the vicinity of the threshold. However, since the BED model uses the actual continuum dipole oscillator strengths, its high- $T$ behavior and the energy distribution of ejected electrons should be more reliable than those of the BEB model. Note that the data by Straub et al. are in excellent agreement with the BED cross section for $T \geqslant 300 \mathrm{eV}$, while the data by Rapp and Englander-Golden for $T>500 \mathrm{eV}$ are slightly higher, a general trend also seen in their cross sections for other targets.

In Fig. 3, the BEB cross section for $\mathrm{O}_{2}$ is compared to the experimental data by Rapp and Englander-Golden, ${ }^{15}$ the

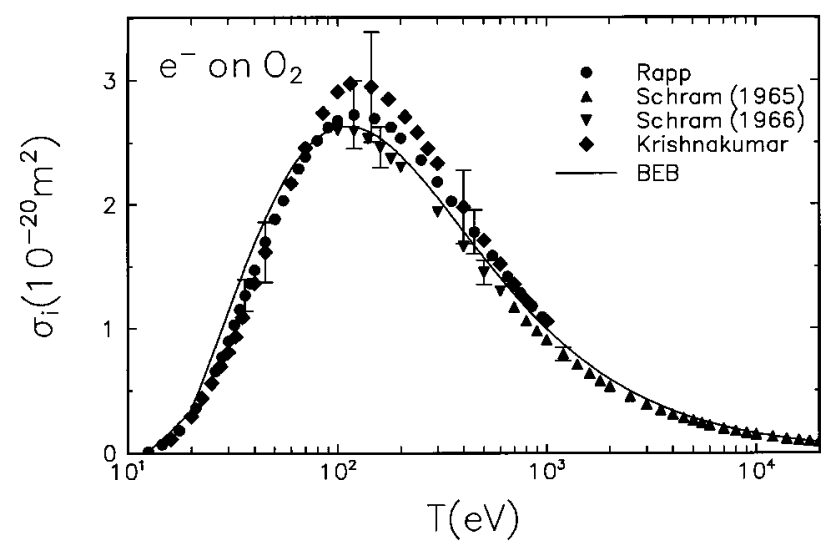

FIG. 3. Comparison of the BEB cross section to experiment for $\mathrm{O}_{2}$. Solid curve, the BEB cross section; circles, experimental data by Rapp and Englander-Golden (Ref. 15); triangles, data by Schram et al. (Ref. 17); inverted triangles, data by Schram et al. (Ref. 18); diamonds, data by Krishnakumar and Srivastava (Ref. 23). 


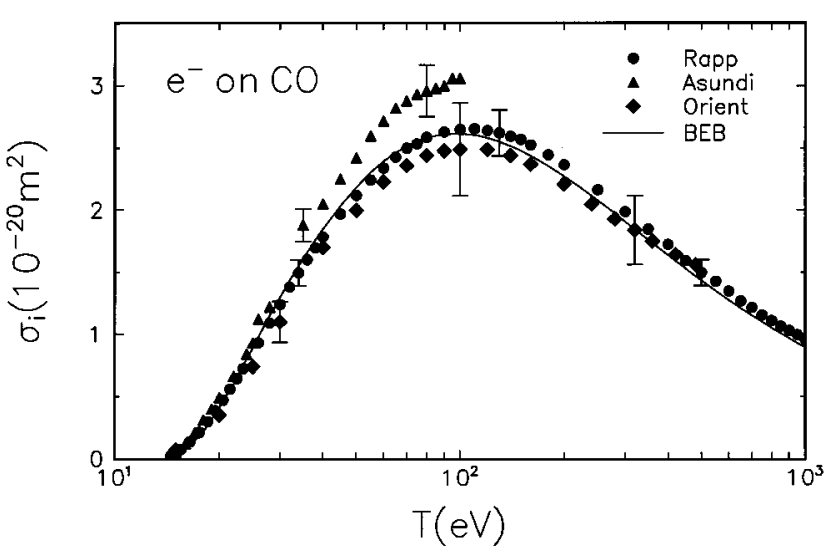

FIG. 4. Comparison of the BEB cross section to experiment for CO. Solid curve, the BEB cross section; circles, experimental data by Rapp and Englander-Golden (Ref. 15); triangles, data by Asundi et al. (Ref. 24); diamonds, data by Orient and Srivastava (Ref. 25).

data by Schram et al., ${ }^{17,18}$ and those by Krishnakumar and Srivastava. ${ }^{23}$ Unlike the case of $\mathrm{H}_{2}$ and $\mathrm{N}_{2}$, the BEB cross section is lower near the peak than the experimental values by Rapp and Englander-Golden. This is the first case in which we used the UHF method for the wave function and took the average of the $\alpha$ and $\beta$ orbital values. The theoretical $B$ values for the outer valence orbitals did not agree well with known experimental values, which we interpret as an indication that a better wave function may be needed.

In Fig. 4, the BEB cross section for CO is compared to the experimental data by Rapp and Englander-Golden, ${ }^{15}$ the data by Asundi et al. ${ }^{24}$ and those by Orient and Srivastava. ${ }^{25}$ The data by Asundi et al. seem to be in clear disagreement not only with other measurements but also with our BEB cross section.

In Fig. 5, the BEB cross section for NO is compared to the experimental data by Rapp and Englander-Golden, ${ }^{15}$ and those by Iga et $_{\text {al. }}{ }^{26}$ The $B$ values of NO require special attention because there are many ionization potentials corre-

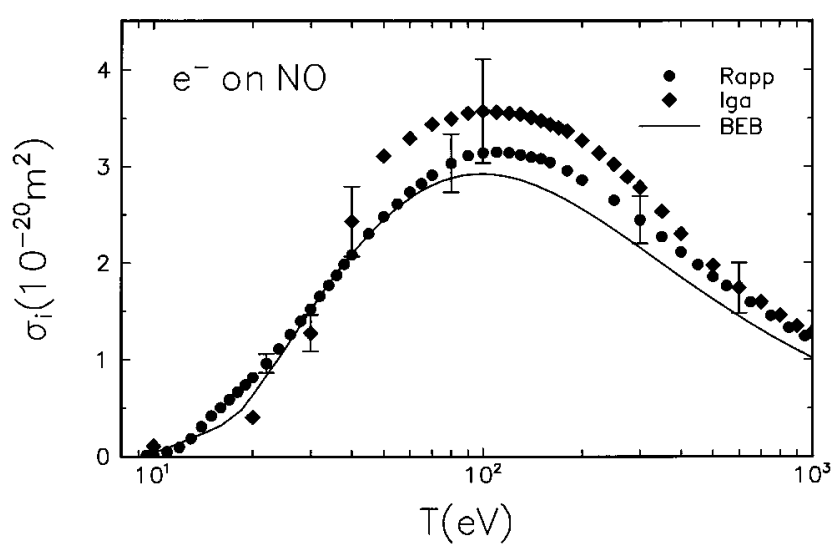

FIG. 5. Comparison of the BEB cross section to experiment for NO. Solid curve, the BEB cross section; circles, experimental data by Rapp and Englander-Golden (Ref. 15); diamonds, data by Iga et al. (Ref. 26).

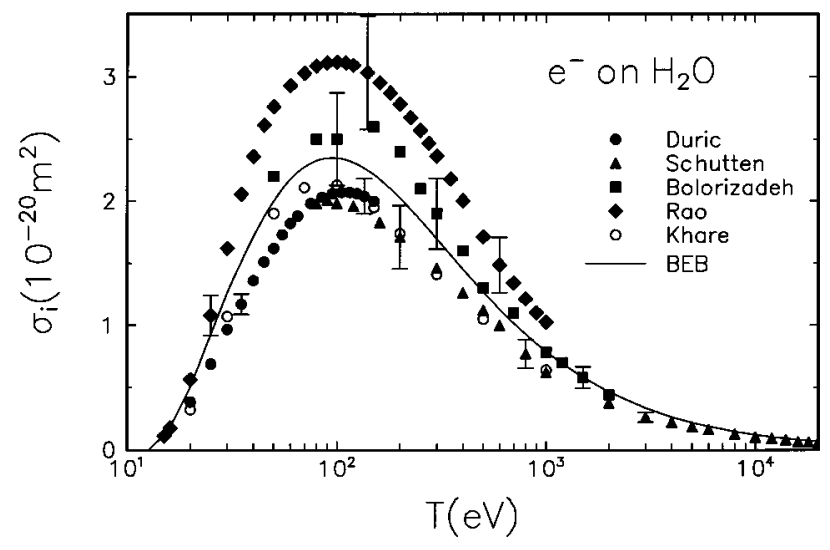

FIG. 6. Comparison of the BEB cross section to experiment for $\mathrm{H}_{2} \mathrm{O}$. Solid curve, the BEB cross section; open circles, semiempirical theory by Khare and Meath (Ref. 5); filled circles, experimental data by Durić et al. (Ref. 29); triangles, data by Schutten et al. (Ref. 31); squares, data by Bolorizadeh and Rudd (Ref. 30); diamonds, data by Rao et al. (Ref. 32).

sponding to many ionic states generated by the ionization of a $5 \sigma$ or $1 \pi$ electron. Among these ionic states, the ${ }^{3} \Pi$ state created by the ionization of a $5 \sigma$ electron dominates the photoionization cross section measured using a photon of $\sim 21 \mathrm{eV}$ in energy. ${ }^{27}$ Although we do not expect the electronimpact ionization cross sections to have the same ratios as those by photoionization, we assumed that the threshold behavior would be similar, and used the experimental values of $B$ for the $5 \sigma$ orbital and the $2 \pi$ orbital in the BEB cross section shown in Fig. 5. The average value of the theoretical orbital energies for the $5 \sigma$ orbital is $18.55 \mathrm{eV}$. The "kink" near $T=20 \mathrm{eV}$ is the artifact of the BEB model because the model cannot account for autoionizing states between the lowest $B$ and $T \sim 20 \mathrm{eV}$ that must have been included in the experimental cross sections. Indeed, the experimental data by Kim et $_{\text {al. }}{ }^{28}$ (not shown in the figure) agree very well with those by Rapp and Englander-Golden for $T \leqslant 40 \mathrm{eV}$, indicating that the experimental data by Iga et al. at $10<T<30 \mathrm{eV}$ are too low.

\section{B. $\mathrm{H}_{2} \mathrm{O}, \mathrm{CO}_{2}$, and $\mathrm{NH}_{3}$}

In Fig. 6, the BEB cross section for $\mathrm{H}_{2} \mathrm{O}$ is compared to the experimental data by Duric et al. ${ }^{29}$ the data by Bolorizadeh and Rudd ${ }^{30}$ the data by Schutten et al.,${ }^{31}$ and the data by Rao et al. ${ }^{32}$ The performance of the BEB cross sections observed in closed-shell diatomic molecules indicates that the peak values of the data by Rao et al. are likely to be too high, and those by Schutten et al. too low. The theoretical cross section by Khare and Meath, ${ }^{5}$ which uses experimental $d f / d W$ and other fitted parameters, is lower than our cross section, but the difference is within the uncertainty of our model, about $\pm 15 \%$, at the cross section peak.

The BEB cross section for $\mathrm{CO}_{2}$ shown in Fig. 7 agrees better with the experimental data by Rapp and Englander-Golden ${ }^{15}$ than the data by Orient and Srivastava ${ }^{25}$ near the peak, though the BEB peak value is within the uncertainty of the data by Orient and Srivastava. The shape of 


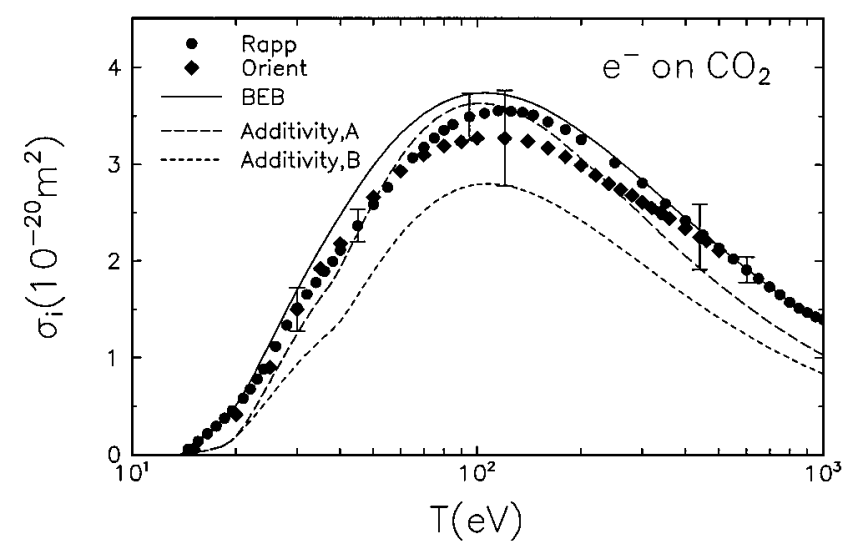

FIG. 7. Comparison of the BEB cross section to experiment for $\mathrm{CO}_{2}$. Solid curve, the BEB cross section; long-dashed curve, semiempirical additivity rule with 32 constants (Ref. 20); short-dashed curve, semiempirical additivity rule with 60 constants (Ref. 20); circles, experimental data by Rapp and Englander-Golden (Ref. 15); triangles, data by Orient and Srivastava (Ref. 25).

the cross section by Orient and Srivastava at $T>200 \mathrm{eV}$ suggests that their values are decreasing too slowly, indicating a systematic trend. One of the two semiclassical cross sections by Margreiter et al. ${ }^{20}$ (marked A in Fig. 7) based on the DM approach, which is an additivity rule discussed in Sec. I, agrees well with experiments except at the very highest incident energies.

As can be seen from Table I, the BEB cross section used 18 constants for $\mathrm{CO}_{2}$, while the DM approach cross sections required 32 constants for curve $\mathrm{A}$ and 60 constants for curve $\mathrm{B}$, including 8 and 15 empirical weighting factors, respectively.

As is shown in Fig. 8, the BEB cross section for $\mathrm{NH}_{3}$ agrees within $10 \%$ with the experimental data by Djuric et $a .^{33}$ and those by Rao and Srivastava ${ }^{34}$ for $T \geqslant 40 \mathrm{eV}$. No error limits were quoted by Djuric et al. As in the case of

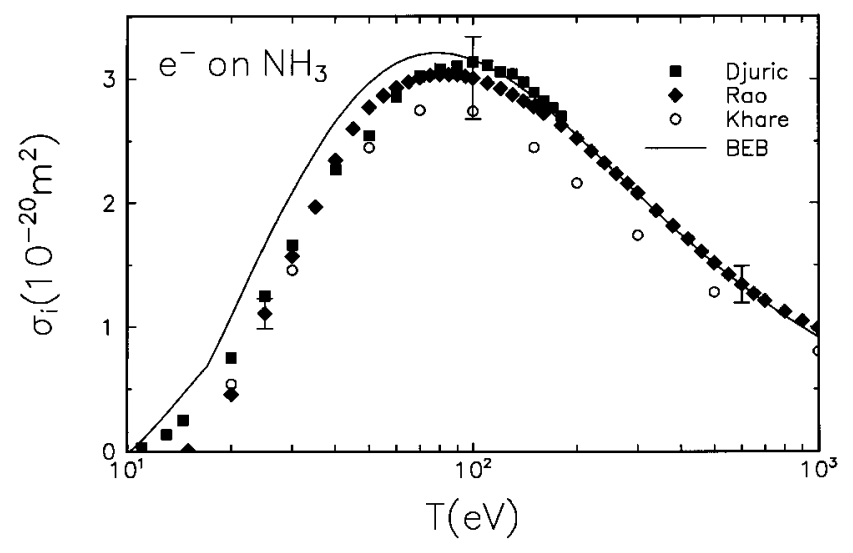

FIG. 8. Comparison of the BEB cross section to experiment for $\mathrm{NH}_{3}$. Solid curve, the BEB cross section; circles, semiempirical theory by Khare and Meath (Ref. 5); squares, experimental data by Djurić et al. (Ref. 33); diamonds, data by Rao and Srivastava (Ref. 34).

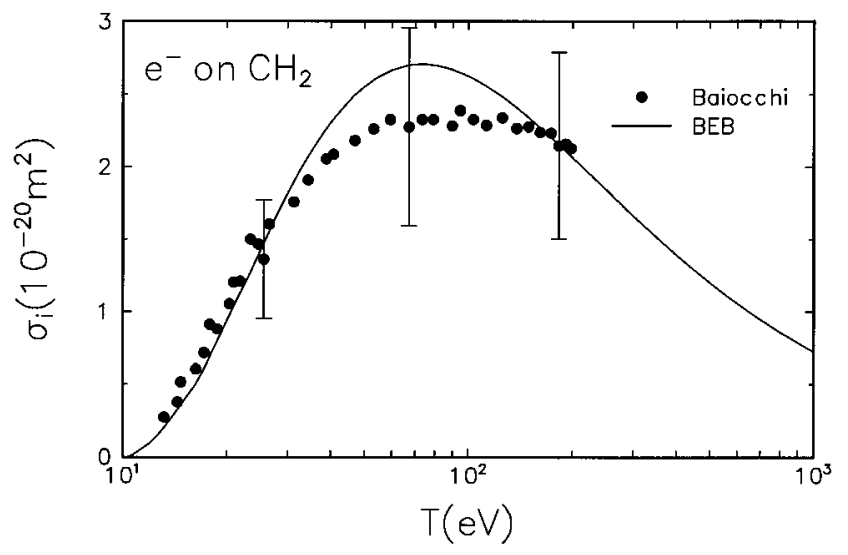

FIG. 9. Comparison of the BEB cross section for $\mathrm{CH}_{2}$ to experimental data for $\mathrm{CD}_{2}$. Solid curve, the BEB cross section; circles, experimental data by Baiocchi et al. (Ref. 36).

$\mathrm{H}_{2} \mathrm{O}$, the peak value of the semiempirical cross section by Khare and Meath ${ }^{5}$ is lower than ours, but the difference is within the uncertainty of our model.

\section{Hydrocarbons}

We found that the BEB model is particularly successful in reproducing known cross sections of hydrocarbons. The DM approach also works well for hydrocarbons. ${ }^{35}$ The BEB cross sections for $\mathrm{CH}_{2}$ and $\mathrm{CH}_{3}$ are compared to the experimental data by Baiocchi et al. ${ }^{36}$ for $\mathrm{CD}_{2}$ (Fig. 9) and $\mathrm{CD}_{3}$ (Fig. 10), respectively. The BEB model is not refined enough to account for the isotope substitution. Although the BEB cross sections for these molecules are in good agreement with the experimental data, Fig. 9 indicates that the experimental data for $\mathrm{CD}_{2}$ are not decreasing as fast as the BEB cross section for $T>150 \mathrm{eV}$. The data by Baiocchi et al. is a lower bound to the total ionization cross section because their experiment did not include the cross sections for the production of atomic ions, $\mathrm{C}^{+}$and $\mathrm{D}^{+}$.

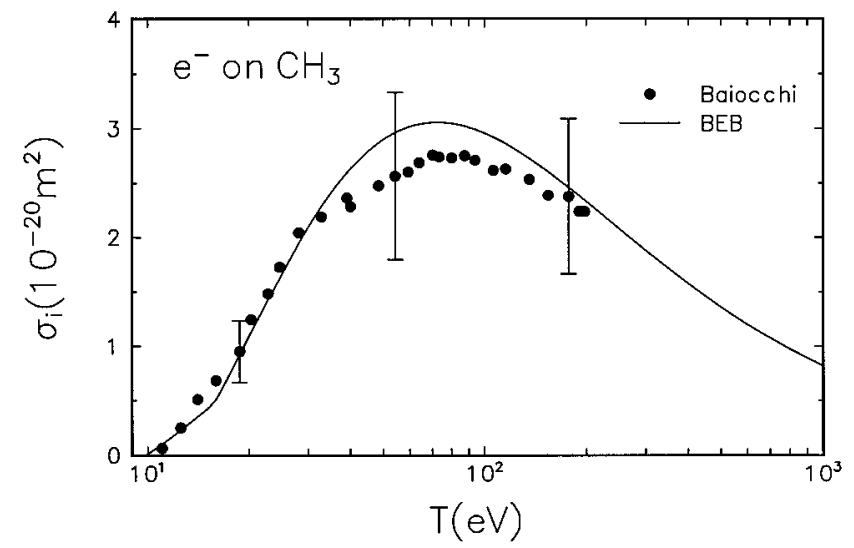

FIG. 10. Comparison of the BEB cross section for $\mathrm{CH}_{3}$ to experimental data for $\mathrm{CD}_{3}$. Solid curve, the $\mathrm{BEB}$ cross section; circles, experimental data by Baiocchi et al. (Ref. 36). 


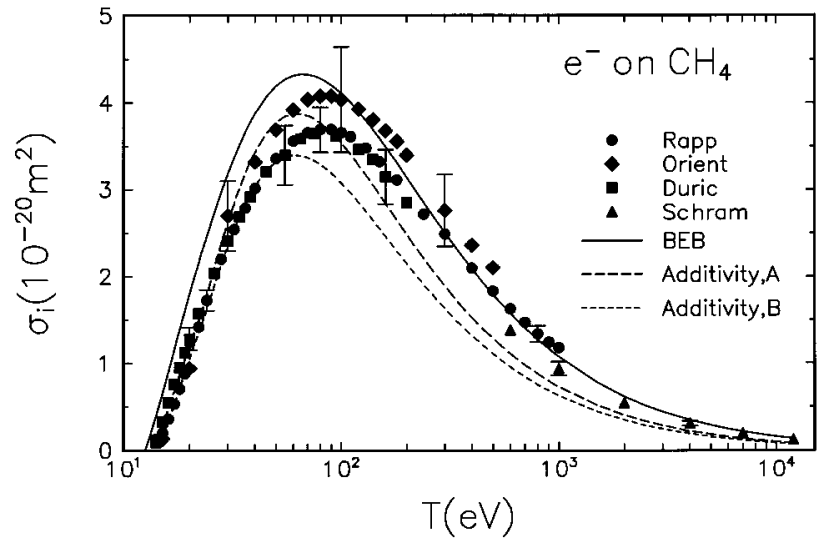

FIG. 11. Comparison of the BEB cross section to experiment for $\mathrm{CH}_{4}$. Solid curve, the BEB cross section; long-dashed curve, semiempirical additivity rule with 12 constants (Ref. 20); short-dashed curve, semiempirical additivity rule with 24 constants (Ref. 20); circles, experimental data by Rapp and Englander-Golden (Ref. 15); diamonds, data by Orient and Srivastava (Ref. 25); squares, data by Durić et al. (Ref. 38); triangles, data by Schram et al. (Ref. 37)

In Fig. 11, the BEB cross section is compared to the experimental data for $\mathrm{CH}_{4}$ by Rapp and Englander-Golden, ${ }^{15}$ the data by Schram et al., ${ }^{37}$ the data by Orient and Srivastava, ${ }^{25}$ and the data by Duric et al. ${ }^{38}$ The BEB cross section is too large between the threshold and the peak. We also included the cross sections generated from the additivity rule ${ }^{20}$ of the DM approach as was done for $\mathrm{CO}_{2}$. For methane, the additivity rule uses 12 constants for curve $\mathrm{A}$ and 24 constants for curve B. The experimental data by Chatham et al. $^{39}$ (not shown in the figure) are very close to those by Durić et al.

The methane molecule has a simple electronic structure (see Table II), and most of its cross section comes from the $1 t_{2}$ valence orbital which has six electrons. The BEB model is vulnerable in this case because any theoretical uncertainty is amplified by the large occupation number of the valence orbital. It may be necessary to use the BED model with reliable continuum dipole oscillator strengths and data from a better molecular wave function to improve the BEB cross section at low $T$.

In Fig. 12, the BEB cross section is compared to the experimental data for $\mathrm{C}_{2} \mathrm{H}_{4}$ by Rapp and Englander-Golden, ${ }^{15}$ and those by Schram et al. ${ }^{37}$ The BEB cross section and the experimental data by Rapp and Englander-Golden seem to disagree near the peak, but the data by Schram et al. are in good agreement with the theory. (Unfortunately, the data by Rapp and Englander-Golden for this molecule stops at $T=145 \mathrm{eV}$ unlike their data on other targets.)

In Fig. 13, the BEB cross section is compared to the experimental data for $\mathrm{C}_{2} \mathrm{H}_{6}$ by Duric et al. ${ }^{38}$ the data by Chatham et al. ${ }^{39}$ the data by Schram et al., ${ }^{37}$ and the data by Grill et al. ${ }^{40}$ Again, the BEB cross section is in good agreement with the experiments. Unlike the case of smaller molecules, the high- $T$ cross section by Schram et al. is now

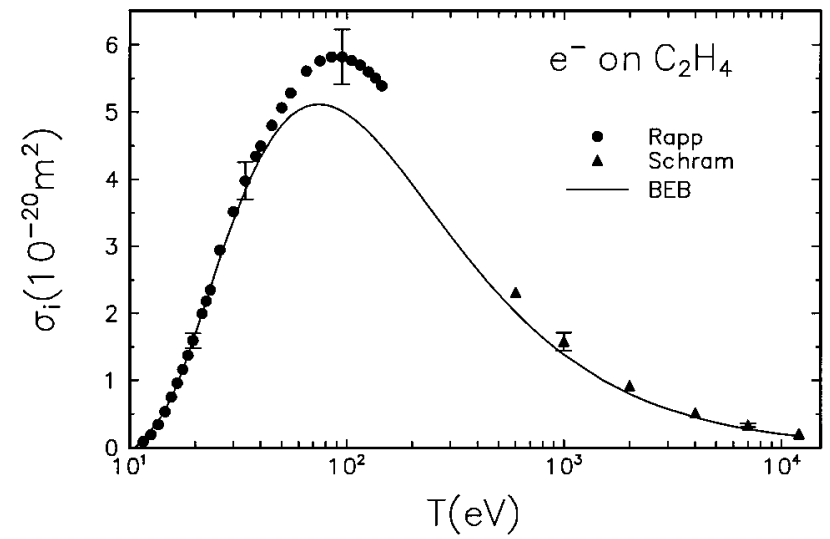

FIG. 12. Comparison of the BEB cross section to experiment for $\mathrm{C}_{2} \mathrm{H}_{4}$. Solid curve, the BEB cross section; circles, experimental data by Rapp and Englander-Golden (Ref. 15); triangles, data by Schram et al. (Ref. 37).

higher than the BEB cross section. The experimental data by Grill et al. agree very well with the BEB cross section at high $T$, making it likely that the data by Schram et al. are too high.

The BEB cross section for $\mathrm{C}_{3} \mathrm{H}_{8}$ in Fig. 14 is in good agreement with the experimental data by Duric et al. ${ }^{38}$ and by Grill et al. ${ }^{41}$ but somewhat lower than those by Schram et al. $^{37}$

In Fig. 15, the BEB cross section is compared to the experimental data for $\mathrm{C}_{6} \mathrm{H}_{6}$ by Schram et al. ${ }^{37}$ which are available only for $T \geqslant 600 \mathrm{eV}$. Although we expect the BEB cross section to be the lower limit to the gross ionization cross section measured by Schram et al., the discrepancy between theory and experiment seems to be too large. Experimental data at lower $T$ are desirable to determine the reliability of the BEB cross section for large molecules. Unlike the case of methane, there is no single molecular orbital that dominates the ionization cross section of benzene, and hence

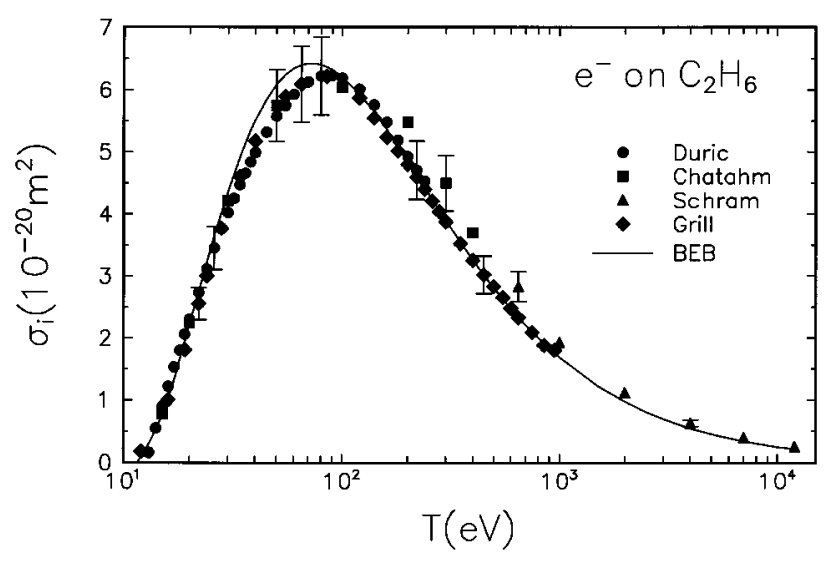

FIG. 13. Comparison of the BEB cross section to experiment for $\mathrm{C}_{2} \mathrm{H}_{6}$. Solid curve, the BEB cross section; circles, experimental data by Duric et al. (Ref. 38); squares, data by Chatham et al. (Ref. 39); triangles, data by Schram et al. (Ref. 37); diamonds, data by Grill et al. (Ref. 40). 


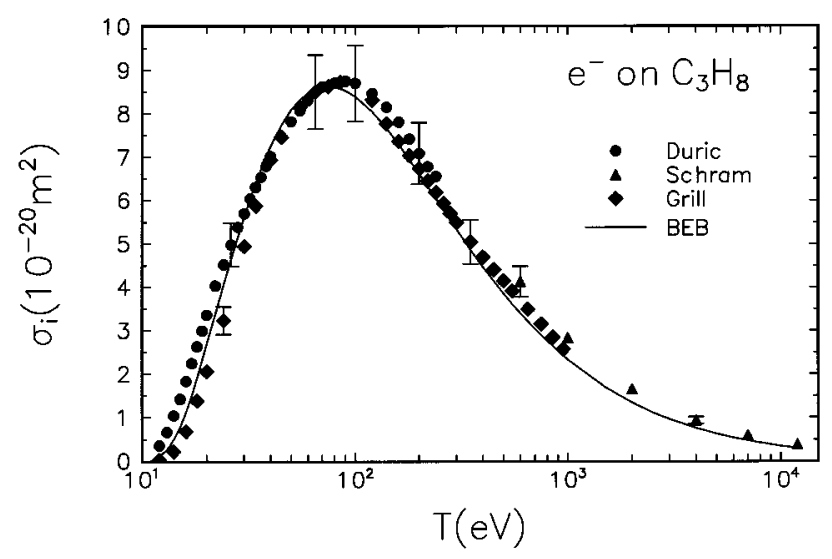

FIG. 14. Comparison of the BEB cross section to experiment for $\mathrm{C}_{3} \mathrm{H}_{8}$. Solid curve, the BEB cross section; circles, experimental data by Duric et al. (Ref. 38); triangles, data by Schram et al. (Ref. 37); diamonds, data by Grill et al. (Ref. 41).

we expect the BEB model cross section at low $T$ to be reliable.

\section{Fluorine compounds}

As was pointed out earlier by Deutsch et al., ${ }^{6}$ fluorine compounds, such as $\mathrm{SiF}_{x}, x=1-3$, exhibit a peculiar behavior: The ionization cross section for a molecule with more fluorine atoms is smaller than the cross section for a molecule with fewer fluorine atoms. ${ }^{42-44}$ This is contrary to the "logic" used in an additivity rule, which expects higher cross sections for molecules with more atoms of the same kind. ${ }^{6}$

The explanation for this "abnormal" behavior is simple; it results from a strong ionic bonding of the $\mathrm{F}$ atoms. The four valence electrons in the $3 s$ and $3 p$ orbitals of $\mathrm{Si}$ have much larger orbital radii than those of the $2 s$ and $2 p$ electrons in F. Hence, the dominant part of the ionization cross section comes from the valence electrons of $\mathrm{Si}$. When one F atom is combined to form $\mathrm{SiF}$, only one valence electron

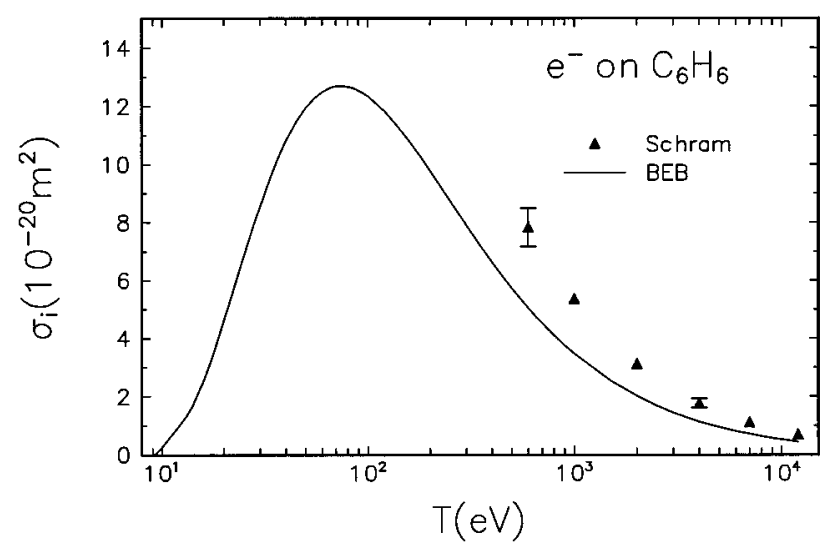

FIG. 15. Comparison of the BEB cross section to experiment for $\mathrm{C}_{6} \mathrm{H}_{6}$. Solid curve, the BEB cross section; triangles, experimental data by Schram et al. (Ref. 37).

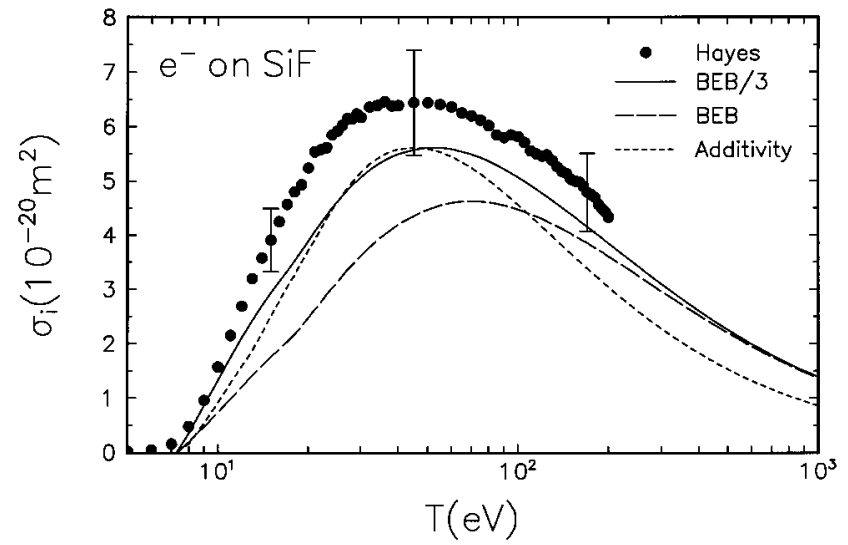

FIG. 16. Comparison of the BEB cross section to experiment for SiF. Solid curve, BEB cross section using modified values of $U$ for the two outermost orbitals; long dashed curve, BEB cross section using unmodified $U$ values; short dashed curve, additivity cross section based on the DM approach by Deutsch et al. (Ref. 6); circles, experimental data for SiF by Hayes et al. (Ref. 42).

from $\mathrm{Si}$ is transferred to $\mathrm{F}$, and the effective ionization cross section comes from the remaining three valence electrons of $\mathrm{Si}$. For $\mathrm{SiF}_{2}$, only two valence electrons of $\mathrm{Si}$ remains, and hence the total ionization cross section is smaller than that of $\mathrm{SiF}$. The same logic explains why $\mathrm{SiF}_{3}$ has smaller ionization cross section than the other two radicals.

So far, we have applied the BEB model to molecules made of light atoms that consisted of only $K$ - and $L$-shell electrons. However, in our experience in applying the BED model to $\mathrm{Ar}, \mathrm{Kr}$, and $\mathrm{Xe}$, we found that the $U$ values of $M$-shell and outer shell electrons had to be divided by their principal quantum numbers to obtain reliable ionization cross sections. Otherwise, BED cross sections were too low at the peak. These outer shell electrons have radial nodes (in atomic orbitals) which make the $U$ values very high. Note that the $U$ values of the molecular orbitals identified with the $M$ electrons of $\mathrm{Si}$ in Table III are more than five times the corresponding values of $B$. These large $U$ values decrease the contributions from the valence electrons, which are usually the dominant ones.

We have applied the same remedy to the BEB cross sections for $\mathrm{SiF}, \mathrm{SiF}_{2}$, and $\mathrm{SiF}_{3}$ by reducing the $U$ values of the valence molecular orbitals clearly identified with the $3 \mathrm{~s}$ and/or $3 p$ electrons of $\mathrm{Si}$ through the Mulliken population analysis. Such orbitals are identified in Table III, and their $U$ values were divided by three as indicated in the table. As expected, the charge density of the two outermost orbitals of $\mathrm{SiF}$ and one valence orbital each in $\mathrm{SiF}_{2}$ and $\mathrm{SiF}_{3}$ are dominantly $(\geqslant 90 \%)$ from Si. For instance, we used $U(7 \sigma)$ $=55.09 / 3=18.36 \mathrm{eV}$, and $U(3 \pi)=34.39 / 3=11.46 \mathrm{eV}$ for $\mathrm{SiF}$.

The BEB cross sections with these modified $U$ values are compared to experimental data for $\mathrm{SiF}$ by Hayes et al., ${ }^{42}$ $\mathrm{SiF}_{2}$ by Shul et al., ${ }^{43}$ and for $\mathrm{SiF}_{3}$ by Hayes et al. ${ }^{44}$ in Figs. 16-18, respectively. The curves marked "BEB/3" represent BEB cross sections with the modified $U$ values, while the 


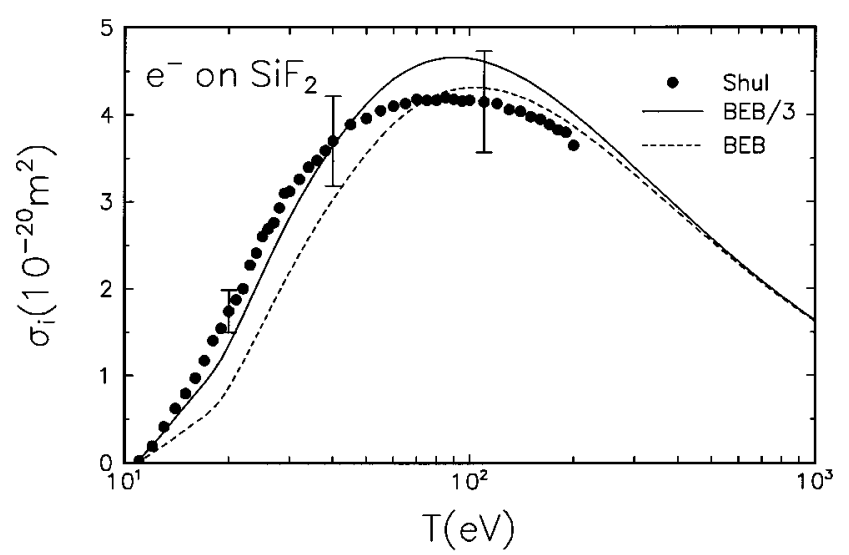

FIG. 17. Comparison of the BEB cross section to experiment for $\mathrm{SiF}_{2}$. Solid curve, BEB cross section using modified value of $U$ for the outermost orbitals; dashed curve, BEB cross section using unmodified $U$ value; circles, experimental data for $\mathrm{SiF}_{2}$ by Shul et al. (Ref. 43).

curves marked "BEB" used the unmodified $U$ values as we did for other molecules in this article.

Even with this modification, the BEB model grossly overestimates the cross section for $\mathrm{SiF}_{3}$ in the peak region. A detailed analysis of the $\mathrm{BEB}$ cross section reveals that the contributions from the extra electrons in $\mathrm{SiF}_{3}$ made its peak cross section comparable to that of $\mathrm{SiF}_{2}$ contrary to the experiment. Since the UHF method tends to produce unreliable $B$ values for outer valence electrons, $\mathrm{SiF}_{3}$ may be a candidate for using data from a more sophisticated wave function. As is shown in Fig. 16, the DM approach ${ }^{6}$ also has difficulty as we do in reproducing the experimental data well.

On the other hand, we find in Fig. 19 that the BEB cross section for $\mathrm{SF}_{6}$ is in reasonable agreement with the experiment by Rapp and Englander-Golden, ${ }^{15}$ probably because all valence electrons on $\mathrm{S}$ are strongly bound to $\mathrm{F}$ atoms, losing their atomic character according to the population analysis.

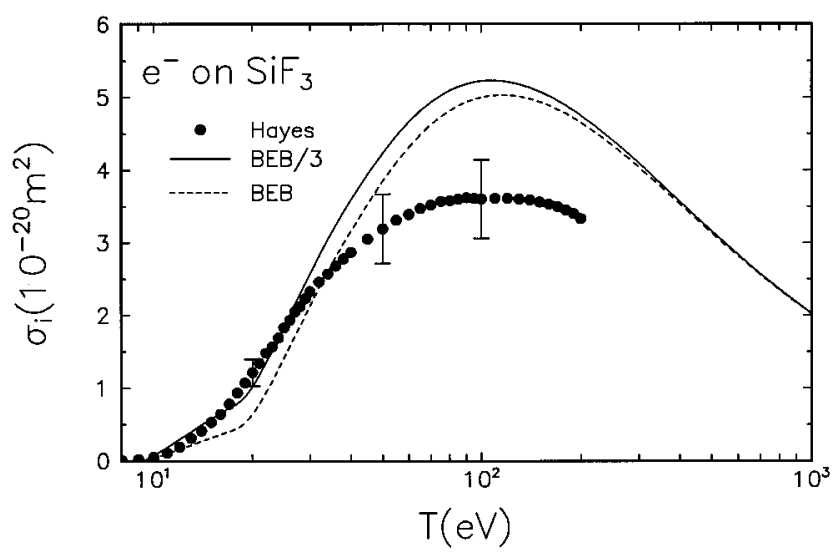

FIG. 18. Comparison of the BEB cross section to experiment for $\mathrm{SiF}_{3}$. Solid curve, BEB cross section using modified value of $U$ for the outermost orbitals; dashed curve, BEB cross section using unmodified $U$ value; circles, experimental data for $\mathrm{SiF}_{3}$ by Hayes et al. (Ref. 44).

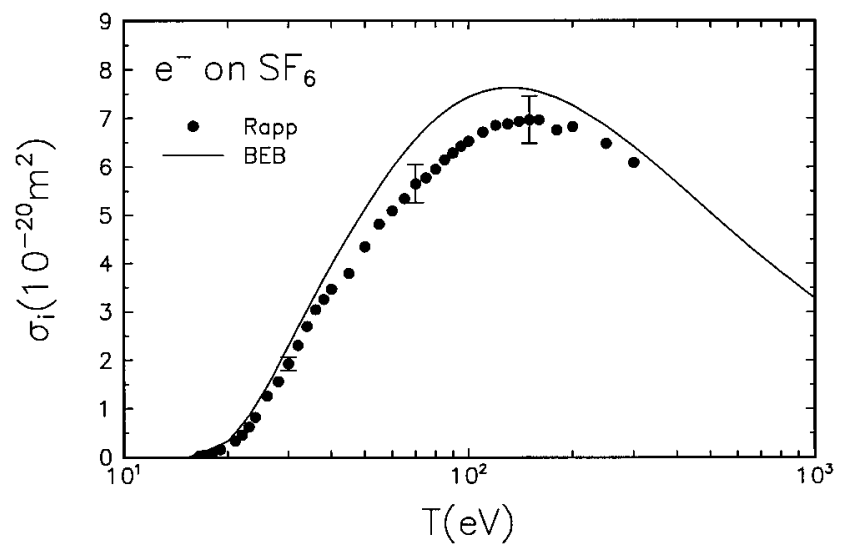

FIG. 19. Comparison of the BEB cross section to experiment for $\mathrm{SF}_{6}$. Solid curve, the BEB cross section; circles, experimental data by Rapp and Englander-Golden (Ref. 15).

Since no valence electron of $\mathrm{SF}_{6}$ retained the $M$-shell characteristics of $\mathrm{S}$, we used unmodified $U$ values for all orbitals.

Ionization cross sections of fluorine compounds such as $\mathrm{BF}_{x}, \mathrm{CF}_{x}$, and $\mathrm{SiF}_{x}$ and similar chlorine compounds will serve as the "acid test" not only for the BEB model but also for any scheme that provides theoretical ionization cross sections.

\section{CONCLUSIONS}

We have demonstrated that the BEB and BED cross sections provide reliable electron-impact total ionization cross sections for a large variety of molecules, except for $\mathrm{SiF}_{3}$, from ionization threshold to high incident energies, $T \sim 10$ $\mathrm{keV}$. The BEB cross section requires only a minimal set of molecular constants for the initial state of the target molecule, which are readily available from public-domain molecular structure codes.

Moreover, the BEB equation consists of simple analytic expressions as functions of the incident energy for each molecular orbital that contributes to the ionization cross section, making the cross sections ideally suited for applications in modeling low-energy plasmas in plasma processing and fusion devices. When appropriate continuum oscillator strengths are available, the BED model provides better energy distribution of ejected electrons (singly differential cross sections) as well as total ionization cross sections. The BEB model uses far fewer constants than the additivity rules known as the DM approach. The latter also requires empirically fitted parameters, while our model has no adjustable parameters. Molecular orbital constants needed to construct BEB cross sections for 19 common molecules have been presented in Tables I-III.

The success of the BEB model on such a wide range of molecules is somewhat surprising, because our experience on atomic ionization cross sections clearly indicated that the BED model with appropriate continuum oscillator strengths was needed for good agreement with experiment. We speculate that the break-up of atomic orbitals to many molecular 
orbitals in a molecule must act as a sort of "averaging" of atomic character and makes the BEB model adequate for most molecules.

Undoubtedly, a simple theory such as the BEB model will require further refinements to expand its application to a wider class of molecules, as we have already seen for fluorine compounds. Chlorine compounds are expected to have similar problems. Meanwhile, we are confident that the BEB model will reliably predict the ionization cross sections of hydrocarbons and other molecules made of light atoms, particularly closed-shell molecules.

BEB cross sections near the ionization threshold are sensitive to the lowest values of $B$ and $U$ used. To insure the proper behavior near the ionization threshold, experimental values of the lowest ionization potential, which are well known for many molecules and radicals, ${ }^{13}$ should be used as the lowest $B$. In some cases, cross sections near the threshold are also likely to be influenced by resonances and autoionization peaks, making it difficult for a simple theory such as the BEB or BED model to be universally effective.

For those who are interested in representing a known cross section by a simple analytic formula, Eq. (5) can be used - for the total ionization cross section, not orbital cross sections-by taking the lowest $B$ to be the first ionization potential, as we did to insure proper ionization threshold, but treating $U$ and $N$ as fitting parameters. For instance, using a higher value of $U$ for the outermost orbital in $\mathrm{CH}_{4}$ and $\mathrm{SF}_{6}$ (while keeping other constants to the values in Tables I-III) will reproduce the experimental cross sections by Rapp and Englander-Golden ${ }^{15}$ in Figs. 11 and 19 to a very high accuracy between the threshold and the peak without significantly altering the high- $T$ part of the BEB cross section.

Work is in progress to extend the BEB model to other molecules of interest to air pollution and plasma chemistry modeling.

\section{ACKNOWLEDGMENTS}

We are grateful to the creators of the GAMESS code (see Ref. 12), without which we could not have carried out this work. We are indebted to Professor M. A. Ali for valuable advice on molecular wave functions, and to Dr. S. K. Srivastava, Professor T. M. Märk, and Professor R. F. Stebbings for providing tables of their experimental data. This work at NIST was supported in part by the Office of Fusion Energy of the U.S. Department of Energy, and at the University of Nebraska-Lincoln by the National Science Foundation Grant No. PHY-9119818.

${ }^{1}$ I. Bray, J. Phys. B 28, L247 (1995).

${ }^{2}$ N. F. Mott, Proc. R. Soc. London Ser. A 126, 259 (1930).

${ }^{3}$ L. Vriens, in Case Studies in Atomic Physics, Vol. 1, edited by E. W. McDaniel and M. R. C. McDowell (North Holland, Amsterdam, 1969), p. 335.

${ }^{4}$ H. Bethe, Ann. Phys. 5, 325 (1930).

${ }^{5}$ S. P. Khare and W. J. Meath, J. Phys. B 20, 2101 (1987), and references therein.

${ }^{6}$ H. Deutsch, C. Cornelissen, L. Cespiva, V. Bonacic-Koutecky, D. Margreiter, and T. D. Märk, Int. J. Mass Spectrom. Ion Processes 129, 43 (1993), and references therein.

${ }^{7}$ H. Deutsch, T. D. Märk, V. Tarnovsky, K. Becker, C. Cornelissen, L.
Cespiva, and V. Bonacic-Koutecky, Int. J. Mass Spectrom. Ion Processes 137, 77 (1994), and references therein.

${ }^{8}$ S. M. Seltzer, in Monte Carlo Transport of Electrons and Photons, edited by T. M. Jenkins, W. R. Nelson, and A. Rindi (Plenum, New York, 1988), p. 81 , and references therein.

${ }^{9}$ M. Gryzinski, Phys. Rev. 138, A305 (1965); 138, A322 (1965); 138, A336 (1965)

${ }^{10}$ Y.-K. Kim and M. E. Rudd, Phys. Rev. A 50, 3954 (1994)

${ }^{11}$ W. Kolos and C. C. J. Roothaan, Rev. Mod. Phys. 32, 205 (1960).

${ }^{12}$ We have used the version of GAMESS described by M. W. Schmidt, K. K. Baldridge, J. A. Boatz, S. T. Elbert, M. S. Gordon, J. H. Jensen, S. Koseki, N. Matsunaga, K. A. Nguyen, S. J. Su, T. L. Windus, M. Dupuis, and J. A. Montgomery, J. Comput. Chem. 14, 1347 (1993).

${ }^{13}$ S. G. Lias, J. F. Liebman, R. D. Levin, and S. A. Kafafi, "NIST Positive Ion Energetics Database, Version 2.0," Standard Reference Database 19A, National Institute of Standards and Technology, Oct. 1993.

${ }^{14}$ M. E. Rudd, Y.-K. Kim, D. H. Madison, and T. J. Gay, Rev. Mod. Phys. 64, 441 (1992).

${ }^{15}$ D. Rapp and P. Englander-Golden, J. Chem. Phys. 43, 1464 (1965).

${ }^{16}$ E. Krishnakumar and S. K. Srivastava, J. Phys. B 27, L251 (1994). See also, E. Krishnakumar and S. K. Srivastava, Abstracts, 16th Int. Conf. on the Physics of Electronic and Atomic Collisions, edited by A. Dalgarno, R. S. Freund, M. S. Lubell, and T. B. Lucatorto (New York, 1989), p. 326.

${ }^{17}$ B. L. Schram, F. J. de Heer, M. J. van der Wiel, and J. Kistenmaker, Physica 31, 94 (1965).

${ }^{18}$ B. L. Schram, H. R. Moustafa, J. Schutten, and F. J. de Heer, Physica 32, 734 (1966).

${ }^{19}$ D. R. Schultz, L. Meng, and R. E. Olsen, J. Phys. B 25, 4601 (1992).

${ }^{20}$ D. Margreiter, H. Deutsch, M. Schmidt, and T. D. Märk, Int. J. Mass Spectrom. Ion Processes 100, 157 (1990).

${ }^{21}$ E. Krishnakumar and S. K. Srivastava, J. Phys. B 23, 1893 (1990).

${ }^{22}$ H. C. Straub, P. Renault, B. G. Lindsay, K. A. Smith, and R. F. Stebbings (to be published).

${ }^{23}$ E. Krishnakumar and S. K. Srivastava, Int. J. Mass. Spectrom. Ion Processes 113, 1 (1992).

${ }^{24}$ R. K. Asundi, J. D. Craggs, and M. V. Kurepa, Proc. Phys. Soc. London 82, 967 (1963).

${ }^{25}$ O. J. Orient and S. K. Srivastava, J. Phys. B 20, 3923 (1987).

${ }^{26}$ I. Iga, M. V. V. S. Rao, and S. K. Srivastava, J. Geophys. Res. (in press).

${ }^{27}$ K. Kimura, S. Katsumata, Y. Achiba, T. Yamazaki, and S. Iwata, Handbook of He I Photoelectron Spectra of Fundamental Organic Molecules (Japan Scientific Societies, Tokyo, 1981), p. 29.

${ }^{28}$ Y. B. Kim, K. Stephan, E. Märk, and T. D. Märk, J. Chem. Phys. 74, 6771 (1981).

${ }^{29}$ N. Lj. Durić, I. M. Čadež, and M. V. Kurepa, Int. J. Mass. Spectrom. Ion Processes 83, R7 (1988).

${ }^{30}$ M. A. Bolorizadeh and M. E. Rudd, Phys. Rev. A 33, 882 (1985).

${ }^{31}$ J. Schutten, F. J. de Heer, H. R. Moustafa, A. J. H. Boerboom, and J. Kistemaker, J. Chem. Phys. 44, 3924 (1966).

${ }^{32}$ M. V. V. S. Rao, I. Iga, and S. K. Srivastava, J. Geophys. Res. (in print).

${ }^{33}$ N. Djurić, D. Belić, M. Kurepa, J. U. Mack, J. Rothleitner, and T. D. Märk, Abstracts, 12th Int. Conf. on the Physics of Atomic and Electronic Collisions, edited by S. Datz (Gatlinburg, 1981), p. 384.

${ }^{34}$ M. V. V. S. Rao and S. K. Srivastava, J. Phys. B 25, 2175 (1992)

${ }^{35}$ H. Deutsch and M. Schmidt, Beitr. Plasmaphys. 24, 475 (1984).

${ }^{36}$ F. A. Baiocchi, R. C. Wetzel, and R. S. Freund, Phys. Rev. Lett. 53, 771 (1984).

${ }^{37}$ B. L. Schram, M. J. van der Wiel, F. J. de Heer, and H. R. Moustafa, J. Chem. Phys. 44, 49 (1966).

${ }^{38}$ N. Durić, I. Čadež, and M. Kurepa, Int. J. Mass Spectrom. Ion Processes 108, R1 (1991).

${ }^{39}$ H. Chatham, D. Hills, R. Robertson, and A. Gallagher, J. Chem. Phys. 81, 1770 (1984).

${ }^{40}$ V. Grill, G. Walder, P. Scheier, M. Krudel, and T. D. Märk, Int. J. Mass Spectrom. Ion Processes 129, 31 (1993).

${ }^{41}$ V. Grill, G. Walder, D. Margreiter, T. Rauth, H. U. Poll, P. Scheier, and T. D. Märk, Z. Phys. D 25, 217 (1993).

${ }^{42}$ T. R. Hayes, R. C. Wetzel, F. A. Baiocchi, and R. S. Freund, J. Chem. Phys. 88, 823 (1988).

${ }^{43}$ R. J. Shul, T. R. Hayes, R. C. Wetzel, F. A. Baiocchi, and R. S. Freund, J. Chem. Phys. 89, 4042 (1988).

${ }^{44}$ T. R. Hayes, R. J. Shul, F. A. Baiocchi, R. C. Wetzel, and R. S. Freund, J. Chem. Phys. 89, 4035 (1988). 